\title{
Rainbow trout discriminate 2-D photographs of conspecifics from distracting stimuli using an innovative operant conditioning device
}

\author{
Aude Kleiber ${ }^{1}$. Claudiane Valotaire ${ }^{1}$ - Amélie Patinote ${ }^{1}$ - Pierre-Lô Sudan ${ }^{1}$ - Guillaume Gourmelen ${ }^{1}$. Cécile Duret ${ }^{1}$. \\ Frédéric Borel ${ }^{1}$ • Leny Legoff ${ }^{1}$ - Manon Peyrafort ${ }^{2} \cdot$ Vanessa Guesdon $^{3}$ • Léa Lansade ${ }^{4}$ Ludovic Calandreau $^{4}$. \\ Violaine Colson ${ }^{1}$ (D)
}

Accepted: 23 November 2020 / Published online: 6 January 2021

(C) The Psychonomic Society, Inc. 2021

\begin{abstract}
Cognitive abilities were studied in rainbow trout, the first continental fish production in Europe. Increasing public concern for the welfare of farmed-fish species highlighted the need for better knowledge of the cognitive status of fish. We trained and tested 15 rainbow trout with an operant conditioning device composed of self-feeders positioned in front of visual stimuli displayed on a screen. The device was coupled with a two-alternative forced-choice (2-AFC) paradigm to test whether rainbow trout can discriminate 2-D photographs of conspecifics (S+) from different visual stimuli (S-). The S- were applied in four stages, the last three stages representing increasing discrimination difficulty: (1) blue shapes; (2) black shape (star); (3) photograph of an object (among a pool of 60); (4) photograph of another fish species (among a pool of 60). Nine fish (out of 15) correctly managed to activate the conditioning device after 30-150 trials. The rainbow trout were able to discriminate images of conspecifics from an abstract shape (five individuals out of five) or objects (four out of five) but not from other fish species. Their ability to learn the category "fish shape" rather than distinguishing between conspecifics and heterospecifics is discussed. The successful visual discrimination task using this complex operant conditioning device is particularly remarkable and novel for this farmed-fish species, and could be exploited to develop cognitive enrichments in future farming systems. This device can also be added to the existing repertoire of testing devices suitable for investigating cognitive abilities in fish.
\end{abstract}

Keywords Operant conditioning · Alternative forced-choice test $\cdot$ Rainbow trout $\cdot$ Conspecific recognition $\cdot$ Object recognition $\cdot$ Categorization

\section{Introduction}

In psychology and biology, cognition refers to the mechanisms by which animals acquire, process, store, and act on information from the environment (Shettleworth, 2009). In

Violaine Colson

violaine.colson@inrae.fr

1 INRAE, LPGP (Laboratoire de Physiologie et Génomique des Poissons), Campus de Beaulieu, 35042 Rennes, France

2 Université de Caen, EthoS (Ethologie animale et humaine), Campus 5, 14000 Caen, France

3 Junia, CASE (Comportement Animal et Systèmes d'Elevage), 59000 Lille, France

4 INRAE, PRC (Physiologie de la Reproduction et des Comportements), CNRS, IFCE, Université Tours, 37380 Nouzilly, France their environment, animals constantly face various situations to be solved. Cognitive abilities thus play a crucial role in their daily life as they enable certain common key functions such as foraging, predator avoidance, mate-choice, navigation, and orientation (Gierszewski, Bleckmann, \& Schluessel, 2013). To date, most of what we know about animals' cognitive skills focuses on higher vertebrates (primates: Sidman et al., 1982; Vonk, 2003; dolphins: Herman, Gory, Hovancik, \& Bradshaw, 1989), but very few comprehensive studies focused on farmed animals and even fewer on farmed-fish species. However, the attribution of cognitive abilities to animals precisely relates to the way in which the animal will be considered and used. Among farmed animals, rainbow trout (Oncorhynchus mykiss) is the first continental fish species to be commercially produced in Europe (FEAP, 2017). Increasing public concern for the welfare of fish species used for food consumption has highlighted the need to better understand the cognitive status of fish. Cognitive processes are 
key components of fish welfare under farming conditions: individuals need to anticipate specific events (food distribution or netting) or to habituate to stressful situations in order to reduce stress triggered by an unpredictable environment (Cañon Jones, Noble, Damsgård \& Pearce, 2012; Colson et al., 2019; Martins et al., 2012). A better knowledge of the rainbow trout's cognitive abilities would help to find ways to improve their welfare, by developing appropriate - speciesspecific - ethological-based tools, such as cognitive enrichments for future farming systems. Cognitive enrichment, also known as occupational or psychological enrichment, represents the possibility for animals to meet moderate challenges by using their cognitive abilities and/or to actively interact with their environment (Manteuffel, Langbein \& Puppe, 2009; Meehan \& Mench, 2007; Oesterwind, Nürnberg, Puppe \& Langbein, 2016). In this way, an animal is offered the possibility to anticipate and/or to control positive or negative events in order to stimulate its cognition by choosing appropriate behavior.

Fish cognition has begun to be investigated over the last few decades, and studies have revealed that many fish species are endowed with impressive learning skills, comparable to those of non-human primates (Bshary, Wickler, \& Fricke, 2002). Certain fish species have episodic-like memory (Ingraham, Anderson, Hurd \& Hamilton, 2016), make transitive inferences about social relationships (Grosenick, Clement \& Fernald, 2007), attend to their own reputation (Bshary \& Grutter, 2006), use tools (Paśko, 2010; Gerullis \& Schuster, 2014; Millot et al., 2014), solve numerical rules (Agrillo \& Bisazza, 2014; DeLong, Barbato, O’Leary \& Wilcox, 2017; Gomez-Laplaza \& Gerlai, 2013), learn concepts (Gierszewski et al., 2013; Goldman \& Shapiro, 1979; Zerbolio $\&$ Royalty, 1983), orient themselves using a map-like representation of their environment (Broglio, Rodriguez, \& Salas, 2003; Rodriguez, Duran, Vargas, Torres, \& Salas, 1994), and recognize conspecifics individually using visual cues (Clark \& Stephenson, 1999; Culumber, 2015; Griffiths \& Magurran, 1999; Johnston \& Dixson, 2017; Roux et al., 2016; Satoh, Tanaka \& Kohda, 2016). However, none of these cognitive abilities - except visual individual recognition (Höjesjö, Axelsson, Dahy, Gustavsson, \& Johnsson, 2015) was demonstrated in rainbow trout. Visual cues are essential for fish when identifying familiar and unfamiliar individuals, engaging in social learning, avoiding predators, foraging, and navigating (Ioannou, Couzin, James, Croft, \& Krause, 2011). Despite a poor visual acuity compared to humans (Ben-Simon, Ben-Shahar, Vasserman, Ben-Tov, \& Segev, 2012; Champ, Wallis, Vorobyev, Siebeck, \& Marshall, 2014; Hester, 1968; Neumeyer, 2003), some teleost fish are able to discriminate between colors (Oncorhynchus mykiss: Luchiari \& Pirhonen, 2008; Danio rerio: Parker et al., 2012), two- (2-D) and threedimensional (3-D) shapes and even illusory shape surfaces (Gnathonemus petersii: Schuster et al., 2002; Pseudotropheus sp.: Schluessel et al., 2012; Pomacentrus amboinensis: Siebeck et al., 2009; Carassius auratus: Wyzisk et al., 2007).

Most of the tests evaluating fish cognitive abilities are discrimination procedures where fish are previously conditioned to associate and recognize a particular stimulus with positive (S+: food reward) or negative (S-: electric shocks) stimuli (Goldman \& Shapiro, 1979; Zerbolio \& Royalty, 1983). The most commonly used test is the alternative forced-choice (AFC) test, which consists of simultaneously presenting different visual stimuli to the subject, one being rewarded. Fish are frequently tested using the 2-AFC test, a two-choice discrimination test (Danio rerio: Bloch et al., 2019; Parker et al., 2012; Pseudotropheus sp.: Schluessel, Fricke, \& Bleckmann, 2012; Xenotoca eiseni: Sovrano \& Bisazza, 2008). To access the discrimination performance, studies generally use discrete operant responses, such as approaching sensors (Siebeck, Litherland, \& Wallis, 2009), time spent near positive stimuli (Ingraham et al., 2016; Kuroda, Mizutani, Cancado, \& Podlesnik, 2017), and passing through a door to enter a rewarded chamber (Agrillo, Piffer, \& Bisazza, 2010; Bloch, Froc, Pontiggia, \& Yamamoto, 2019; Gomez-Laplaza, Díaz-Sotelo, \& Gerlai, 2018; Mueller \& Neuhauss, 2012; Parker et al., 2012; von der Emde \& Fetz, 2007; Wyzisk \& Neumeyer, 2007), but no studies used the ability of the fish to operate a lever as an active discrete operant response. This last method - also called instrumental conditioning procedure - allows recording the subject's answer with accuracy, particularly when the setup is automated. Moreover, instrumental learning involves a complete panel of cognitive abilities, such as spatial orientation, object recognition, and temporal association of environmental cues (Jurado-Parras et al., 2013), and thus represents an opportunity for investigating cognitive abilities, still little studied in the target fish species chosen for the present experiment, i.e., the rainbow trout.

If we presume that rainbow trout's vision is comparable to that of most of teleost fish species as discussed above, we do not know to which extent they are capable discriminating accurately between conspecifics and any other objects or living animals present in their environment or if they rather form categories on the basis of shared shapes, as shown in the African cichlid Pseudotropheus sp. (Schluessel et al., 2012). This capacity to generalize visual items using categories gives individuals a certain degree of flexibility to react appropriately in case of danger (categories "predator," "shelter"), while hunting (category "prey"), or to ensure positive outcomes (category "reward"), and provide them with stability and safety in a context of changing environments (Franks, 2018).

The aim of this study was to explore the cognitive skills of a farmed fish species by (1) using an original operant conditioning automated device composed of self-feeders, in order to determine (2) whether rainbow trout, the first European continental fish production, are able to solve a simple 2-AFC task, and (3) whether they can recognize conspecifics specifically, or if they rather generalize learned photographs of 
conspecifics to any other fish species sharing a similar shape. For this purpose, we used a pool of four recurrent photographs of trout conspecifics as S+. After a first stage using four different blue shapes as S- which failed in eliciting correct conditioned responses, we then used distracting stimuli as S- according to three stages with increasing discrimination difficulty: stage 2: one black shape (star); stage 3: photograph of an object (among a pool of 60); stage 4: photograph of another fish species (among a pool of 60).

\section{Materials and methods}

\section{Ethics statement}

All experimental procedures used in this study were carried out in strict accordance with the European Directive 2010/63/EU on the protection of animals used for scientific purposes. They have been approved by the Rennes Ethics Committee for Animal Experimentation and have received authorization from the French Ministry of Education, Research and Innovation under agreement number $201806050954214-$ V1-APAFIS\#15361.

\section{Experimental animals}

Female triploid rainbow trout (Oncorhynchus mykiss) were obtained from fertilizations performed at INRA-PEIMA facilities (Sizun, France). Eggs were transferred between 1 and $20 \mathrm{~h}$ post-fertilization, and fish from these eggs were reared at the INRA-LPGP experimental facilities (Rennes, France). Between 50 and 180 days postfertilization, fish were reared in two tanks (72 L: $55 \times 45 \times 29$ $\mathrm{cm}$ ) containing 40 fish each. The density was always below $20 \mathrm{~kg} /$ $\mathrm{m}^{3}$. Tanks were supplied with plants, rocks, and PVC pipes since environmental enrichment is known to enhance cognitive capacities in fish (Salvanes et al., 2013; Strand et al., 2010). At 5 months, 15 fish ( $8 \mathrm{~cm}$ mean length) were randomly chosen and were identified with a PIT tag $(1.4 \times 8 \mathrm{~mm}$; Tiny, Biolog-id, France $)$ implanted above the dorsal fin under anesthesia ( $50 \mathrm{mg} / \mathrm{L}$ tricaine). The 15 fish were housed together in a 336-L tank $(102.5 \times 102.5 \times$ $32 \mathrm{~cm})$ at $12 \pm 0.2^{\circ} \mathrm{C}$ under a 12:12 $\mathrm{h}$ artificial photoperiod. Fish were fed daily at the end of the experiments with extruded and commercial flowing extruded pellets (39\% protein and $24 \%$ lipid, 0.5-mm pellets, Le Gouessant, France). The feeding rate, composition, and diameter of the pellets was regularly adapted according to the fish growth rate. Experiments started after a 2-week acclimation period in the new rearing tank.

\section{Apparatus}

Subjects were tested individually in a 779-L test tank $(104 \times$ $100.5 \times 74.5 \mathrm{~cm}$ ) with the walls painted dark gray (RAL code: 7005). A transparent Plexiglas window $(63 \times 34 \mathrm{~cm})$ included in the tank wall allowed the fish to see the screen placed behind it (Fig. 1). The screen used in this study was an LCD Dell REV A00 (17 in.: $60 \times 34 \mathrm{~cm})$ and displayed visual stimuli. To prevent any distractions, disruptions, or reflections from the screen, a dark sheet was laid behind the screen. Two (or three) self-feeders (removable if needed) were placed in the upper part, and the triggers were $4 \mathrm{~cm}$ from the window and submerged at approximately $9 \mathrm{~cm}$ depth in the water. Fish could reach the pressure needed by the trigger by doing a $5-6^{\circ}$ tilt angle with any part of their body. Once the angle was reached, an electronic microchip located in the cylinder validated the answer. Self-feeders were connected to an automatic food distributor (Imetronic $\AA$, France) delivering coated appetitive pellets (Diana Aqua, France). Their diameter was changed according to fish weight and pellet granulometric recommendations. When a correct answer was recorded, two pellets were released into a funnel connected to a $1.5-\mathrm{cm}$ plastic tube and then fell into the water in the middle of the tank at approximately $30 \mathrm{~cm}$ from the screen. Each reward corresponded to approximately $1 / 12$ th of the fish daily food intake. A camera placed above the experimental device (Samsung SCB-3000PH) and connected to a monitor (EverFocus Colour TFT LCD monitor) permitted the control of the entire real-time experiment at distance without disturbing trout behavior. A GoPro (HERO session) was introduced into the tank once during each stage to capture sequences for illustration purposes only (Fig. 1).

Each experiment was managed by the "POLY M2S" software (Imetronic $®$ ), which allows users to choose the type of experiment, the number of trials, the intertrial interval (ITI), the different stimuli, and their position on the screen. The software also ensures that stimuli do not appear more than two consecutive times at the same position to prevent any side bias. All stimulus sequences were randomly presented (see General procedure).

\section{General procedure}

Fish were never tested more than once a day (one session per day). Experiments were undertaken between 9:00 and 15:00 and the fish were all fed at the same time at the end of the last session, resulting in an overnight food deprivation period varying from $18 \mathrm{~h}$ to $24 \mathrm{~h}$, depending on fish running order.

\section{Training to activate triggers}

Fifteen trout were initially trained to become acclimated to the apparatus and activate the self-feeder triggers. For this habituation phase, three triggers (instead of two for the subsequent stages) were submerged $9 \mathrm{~cm}$ in order to increase their chance of being activated. At the fish entrance in the test area, a food reward was provided to make them associate netting as a positive event. During this habituation 


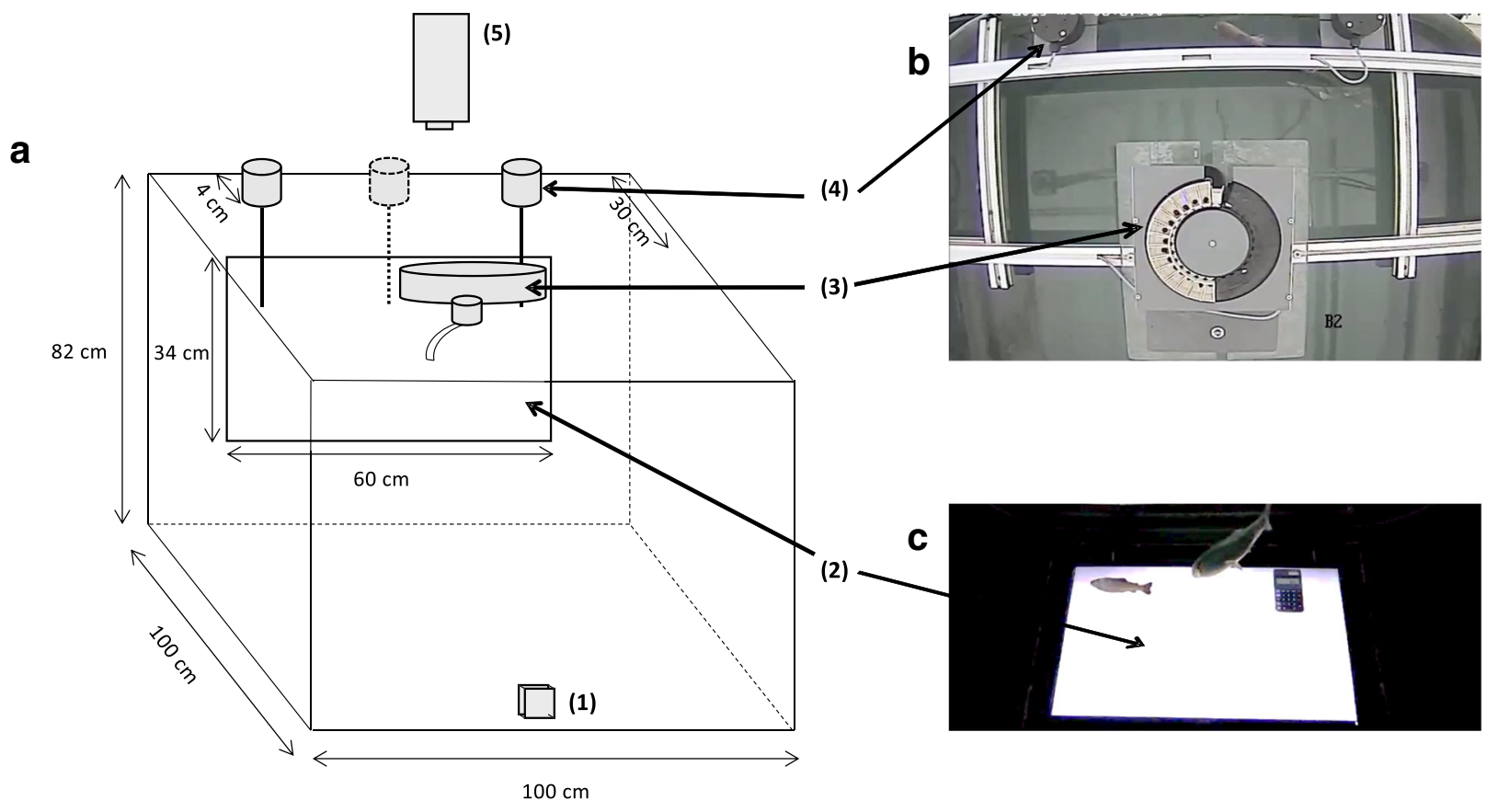

$\begin{array}{lllll}\text { 1) Gopro } & \text { 2) Transparent Plexiglas window } & \text { 3) Food distribution wheel } & \text { 4) Self-feeders (one being removable) 5) Top-view camera }\end{array}$

Fig. 1 a Experimental tank used for the two-alternative forced-choice test task; $\mathbf{b}$ camera view from above; $\mathbf{c}$ GoPro view from inside the tank while stimuli from stage 3 were displayed (here, a calculator as S-)

phase, the monitor displayed a gray screen (RGB code: \#EEECEC) to reduce contrast with the tank color, and the fish received a food reward, regardless of the trigger activated as they were all the right choice. We tested the fish once a day (one session per day), 5 days a week. The first session consisted of five trials of $10 \mathrm{~min}$ each. If a trigger was activated before a 10min delay, the fish received a reward, and the screen turned green (RGB code: \#0F790F) for $60 \mathrm{~s}$, which acts as a secondary positive reinforcement (Luchiari \& Pirhonen, 2008; Zerbolio \& Royalty, 1983). After that, a 10-s ITI with a gray screen occurred, and a new trial began. If the fish did not activate the trigger within the 10-min delay (cut-off), a food reward was still given, and a new trial started after the 10 -s ITI.

In the following sessions, the procedure was the same as in session 1 , but each session included ten trials of 10 min each, and no more reward was given after a cut-off. After each session, the trout was netted and joined its conspecifics. Fish were tested randomly. Individuals were considered to have successfully learned to activate the triggers once they activated one of them seven times (or more) over the ten trials in three consecutive sessions ( $\geq 70 \%$; binomial: $\mathrm{P}<0.001, \mathrm{~N}=$ 30 trials), ensuring the correct application of the operant conditioning device for all subsequent stages. Nine individuals (out of 15) correctly learned trigger activation (see Results section).

\section{Pretraining to activate a trigger associated with a stimulus}

The nine remaining subjects were trained to associate a trigger with the presence of one unique stimulus of different shapes and different colors ( $\mathrm{S}+$ ) displayed behind it on the screen for a total of 540 trials before starting the 2-AFC test. From this exercise until the end of the experiment, only two triggers were available, one being rewarded (right or left position). The S+ never appeared more than twice consecutively on the same side. In the case of correct answers, the fish received a reward, and the screen turned green for $60 \mathrm{~s}$. If the incorrect trigger was activated, no food reward was given, and a red screen appeared (RGB code: \#E90808) for $60 \mathrm{~s}$, followed by a 10-s ITI before starting a new trial. This color acted as a positive punishment to enhance learning. After a cut-off, no food reward was given, and a new trial started after the ITI. Ten trials of 10 min each were conducted per session. An individual was considered to have successfully learned the task once $\mathrm{S}+$ selection was significantly different from chance (50\%) for two consecutive sessions ( $\geq 80 \%$; binomial: $\mathrm{P}=$ $0.012, \mathrm{~N}=20$ trials).

\section{Two-alternative forced-choice test (2-AFC) with photographs of conspecifics as $\mathrm{S}+$}

Only five of the nine individuals were used for the following tasks because one fish died and three individuals stopped being motivated, performing only cut-offs. The 2-AFC test 
consists of presenting two contrasting stimuli simultaneously, one being rewarded (right or left position) (Fig. 2). S+ represented a photograph of trout taken from a pool of four recurrent photographs of unknown conspecifics held in another tank (see Table 1 for the detail of the stimuli used). We chose photographs of conspecifics as biologically relevant stimuli since we know that rainbow trout are able to recognize conspecifics individually using visual cues (Höjesjö et al., 2015; Johnsson \& Åkerman, 1998). The four S+ photographs were presented in equal numbers across the sessions, in a randomized order. A distracting stimulus (S-) was presented at $40 \mathrm{~cm}$ (horizontally) from the $\mathrm{S}+$. The $\mathrm{S}$ - were: stage 1 - four different blue (RGB code: \#1E90FF) shapes (star, triangle, square or circle); stage 2 - a unique black shape (star); stage 3 - a photograph of an object (among a pool of 60 objects); and stage 4 a photograph of another fish species (from a pool of 60 photographs). Blue shapes were first chosen as $\mathrm{S}$ - in stage 1 since we presumed that shapes with an attractive color would counteract the possible natural preference for a biologically relevant stimulus as S+ (Oliveira, Silveira, Chacon \& Luchiari, 2015; Roy Suriyampola, Flores, López, Hickey, Bhat, \& Martins, 2019). Indeed, the color blue has been shown to be preferred (along with green) when given the choice to rainbow trout (Luchiari \& Pirhonen, 2008; Maia, Ferguson, Volpato, \& Braithwaite, 2017). In stage 2, we reduced to one the number of shapes used as $\mathrm{S}$ - because trout failed with four shapes as $\mathrm{S}$ - in stage 1 , and other species succeeded when using only one or two shapes as S- for a similar study design (Gabor \& Gerken, 2012; Langbein, Nurnberg, \& Manteuffel, 2004; Schluessel et al., 2012). Additionally in stage $2, \mathrm{~S}$ - was chosen in a dark color (black) to increase the visual contrast between stimuli. The pool of objects used as S- in stage 3 allowed us to test whether trout only learned to avoid the black star in stage 2 or, conversely, to associate the photograph of a conspecific with a reward. Fish performed these stages in this order and moved on to the next stage as soon as they validated the learning criterion or if they reached the initially chosen maximum number of sessions. A maximum number of 30 sessions was established for stages 1-3, and ten sessions was specified for stage 4 (other fish species). Stage 4 assessed the ability of the fish to discriminate the recurrent photographs of conspecifics from other fish species or, on the contrary, to generalize the learned recurrent stimuli to other stimuli sharing similar features (fish shape). As the instruction given in stage 4 (discriminating between two images of fish) was considered to be ambiguous, we chose ten sessions as a maximum, because more than ten sessions to complete could have cancelled the initial positive association of an image of a trout with a reward and could have acted as a form of reversal learning (Kuroda et al., 2017; Lucon-Xiccato \& Bisazza, 2014). The S+ and S- never appeared more than twice consecutively on the same side. In the case of correct answers, the fish received a reward, and the screen turned green for $60 \mathrm{~s}$. If the incorrect trigger was activated, no food reward was given, and a red screen appeared for $60 \mathrm{~s}$, followed by a 10-s ITI before starting a new trial. After a cut-off (no answer within $10 \mathrm{~min}$ ), no food reward was given, and a new trial started after the ITI. For all stages, each session included 12 trials of 10 min each. An individual was considered to have successfully learned the task once an $\mathrm{S}+$ selection was significantly different from chance $(50 \%)$ for two consecutive sessions $(\geq 75 \%$; binomial: $\mathrm{P}=0.011, \mathrm{~N}=24$ trials).

If the maximum number of sessions was reached by the subject without validating the learning criteria, we repeated stage 2 (black shape as S-) - the initial successful stage of the paradigm - as a control to ensure that the overall performance of the trained 2-AFC task did not decline with time or difficulty, as shown in other studies (archerfish: Newport, Wallis, Temple, \& Siebeck, 2013). Therefore, we used 3 steps for stage 2 (Fig. 2). To prevent any side bias in the first learning exercise (stage 2), a correction trial (CT) was implemented in the case of an incorrect response using the same procedure as described in Knolle, Goncalves, and Morton (2017). This allowed reusing the exact same set of stimuli in the experiment until a correct response was made. However, in subsequent stages, no CT was performed since these stages (objects and other species as S-) were not training stages but testing stages with increasing difficulty. Thus, if a side bias was observed in stages 3 and 4, only a forced test was conducted to eliminate it. This test consisted of a unique $\mathrm{S}+$ presentation (one of the four photographs of conspecifics randomly presented) on the screen on the side opposite from the preferred side until the fish chose the initially non-preferred side in three consecutive trials. No S- was presented in the forced test. From stage 2, the triggers were raised so that they were immersed only $5.5 \mathrm{~cm}$ below the water surface (instead of $9 \mathrm{~cm}$ ) to prevent involuntary activations.

\section{Stimuli}

All stimuli were two-dimensional (2-D) pictures created using Microsoft PowerPoint and ImageJ (Table 1). Images used as positive stimuli were photographs of four juvenile female triploid rainbow trout unfamiliar to the tested trout. The different $\mathrm{S}+$ images used in pretraining (different shapes of different colors) and the S- images (four blue shapes and a black star) used in stages 1 and 2 were created using PowerPoint. During pretraining, 26 different shapes of nine different colors were used randomly, but fish were never exposed to images of fish, objects, or black shapes. For stages 3 and 4, the images used as negative stimuli were all taken from the internet. Stimuli were all stretched according to the PowerPoint slide size $(25.4 \times 19.05 \mathrm{~cm})$ and were individually saved in png format. Then, a macro from ImageJ was 

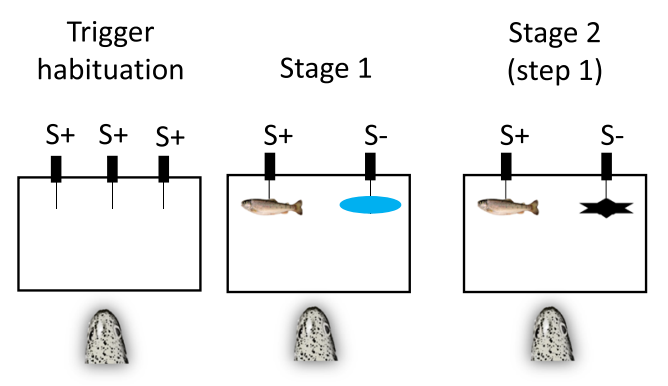

Fig. 2 Successive exercises performed by fish in the two-alternative forced-choice test task after trigger habituation. $S+$ rewarded stimulus; $S$ - examples of unrewarded stimuli. Stage 1: blue shapes as S-; Stage 2

run to adjust their brightness. On the screen, each stimulus appeared at a size of $14.5 \times 11 \mathrm{~cm}$.

\section{Statistical analyses}

All tests and graphs were performed using the 1.2.1335 version of RStudio and were plotted using the packages ggplot2, ggthemes, ggsignif, and effects.

Daily discrimination rates shown by each subject are described graphically as the percent of correct responses per session.

Learning criteria were considered significantly different from chance by using binomial tests. The chance level was $50 \%$ in the case of two triggers.

The percentages of correct responses for each trout during the first session and last session (within each stage) were compared with paired Wilcoxon nonparametric tests.

Eventual preference (proportion of correct responses) for any S+ (four images of trout) and any S- (60 images of objects and 60 of other fish species) was analyzed by
Stage 2

(step 2)

Stage 2

(step 3) 


\section{Results}

\section{Training to activate triggers}

Nine of the 15 trout initially trained to activate the triggers reached the learning criterion of $70 \%$ correct responses in three consecutive sessions (binomial: $\mathrm{P}<0.001, \mathrm{~N}=30$ trials) after three to 15 sessions. The trout that reached the criterion after only three sessions is the one that unfortunately died during the experiment and is thus not further included in the results, which take into account only the five remaining fish. One fish never activated a trigger. The others sometimes activated a trigger, but none were able to reach an accuracy above the level of chance.

\section{Pretraining to activate a trigger associated with a stimulus}

After a total of 540 trials, none of the nine fish reached the learning criterion of $80 \%$ in two consecutive sessions when using shapes of different colors as S+ (binomial: $\mathrm{P}>$ $0.05, \mathrm{~N}=20$ trials).

\section{2-AFC with photographs of conspecifics as S+}

For all stages, no differences in selection frequency were found among the four different conspecific photographs presented as $\mathrm{S}+$ when analyzing the different linear models (GLMs: P > 0.05). Similarly, no preference or aversion was found for any images of objects (stage 3 ) or other fish species (stage 4) presented as $\mathrm{S}-(\mathrm{P}>0.05)$. We did not find any (right or left) side bias at any stage (Wilcoxon: $\mathrm{V}=10, \mathrm{P}>0.05$ ).

\section{Stage 1: Blue shapes as S-}

The first stage of the 2-AFC test consisted of displaying four blue shapes as S- and four recurrent photographs of conspecifics as S+. Even though the scores were mainly at or above the level of chance, none of the five individuals were able to meet the learning criterion in 30 completed sessions (Fig. 3). Trout No. 2 died before completing the 30 sessions and we thus replaced it in the remaining stages by Trout No. 2 bis, after it succeeded in the habituation phase (Table 2).

\section{Stage 2: Black shape as S-}

The learning criterion of $75 \%$ in two consecutive sessions was reached by the five tested individuals (stage 2-step 1, Fig. 3). The trout needed between seven and 14 sessions (84-168 trials) to reach the criterion (Table 2).

Only trout No. 4 was subjected to stage 2-step 2 since it failed in stage 3 (objects as $\mathrm{S}$-). The learning criterion was again reached by this trout after nine sessions $(\mathrm{P}=0.006, \mathrm{~N}$
$=24$ trials) (Fig. 3). The five individuals passed stage 2-step 3 since they all failed in stage 4 (species as S-), and they all again met the learning criterion in two to 15 sessions, except trout No. 5 (maximum of 30 sessions reached).

\section{Stage 3: Objects as S-}

When displaying objects as distracting stimuli, four individuals (out of five) reached a statistically significant $\mathrm{S}+$ selection frequency ( $\geq 75 \%$ correct responses in two consecutive sessions) in two to 12 sessions (binomial: $\mathrm{P}<0.01, \mathrm{~N}=24$ trials) (Fig. 3, Table 2). Trout No. 4 reached the maximum number of 30 sessions without meeting the learning criterion. This trout reached the $75 \%$ correct response level only once (session 13) (Table 2).

\section{Stage 4: Other fish species as S-}

None of the five individuals was able to reach the learning criterion within the maximum of ten sessions established for this stage (Fig. 3). Most of the scores were at or below chance level (Table 2). Trout No. 1 twice reached $75 \%$ or more correct responses but in nonconsecutive sessions (sessions 3 and 9), and trout No. 4 met the criterion only once (session 8 ) (Table 2).

\section{Comparison between first and last session within each stage}

We compared the percentage of correct responses between the first session and the last session for each stage for the five trout tested in the 2-AFC task (Fig. 4). The results indicate a significant increase between the first and last sessions for the trigger habituation stage (Wilcoxon: $\mathrm{V}=0, \mathrm{P}=0.031$, $\mathrm{N}=5$ individuals), for stage 2 (trout as $\mathrm{S}+$ and black shape as $\mathrm{S}-)$, step $1(\mathrm{~V}=0, \mathrm{P}=0.029)$ and for stage 3 (trout as $\mathrm{S}+$ and objects as $\mathrm{S}-)(\mathrm{V}=0, \mathrm{P}=0.029)$. No differences were found for stage 1 (trout as $\mathrm{S}+$ and blue shapes as $\mathrm{S}-)(\mathrm{V}=9, \mathrm{P}=0.812)$ and stage 4 (trout as $\mathrm{S}+$ and other fish species as $\mathrm{S}-)(\mathrm{V}=8, \mathrm{P}=$ 0.607).

We found a significant effect of fish running order on the percentage of correct responses for stage 4 only $\left(\mathrm{LM}: \mathrm{F}_{4,45}=\right.$ $2.90, \mathrm{P}<0.05)$. Fish netted in fifth position had poorer results than those netted in the first (Tukey test: $\mathrm{P}=0.027$ ) and third positions $(\mathrm{P}=0.017)$.

\section{Discussion}

Here, we investigated the capacity of rainbow trout to solve a two-choice discrimination task by using an operant conditioning device. Fish used the operant conditioning apparatus successfully, and we showed that they were able to discriminate between recurrent 2-D photographs of conspecifics and either 


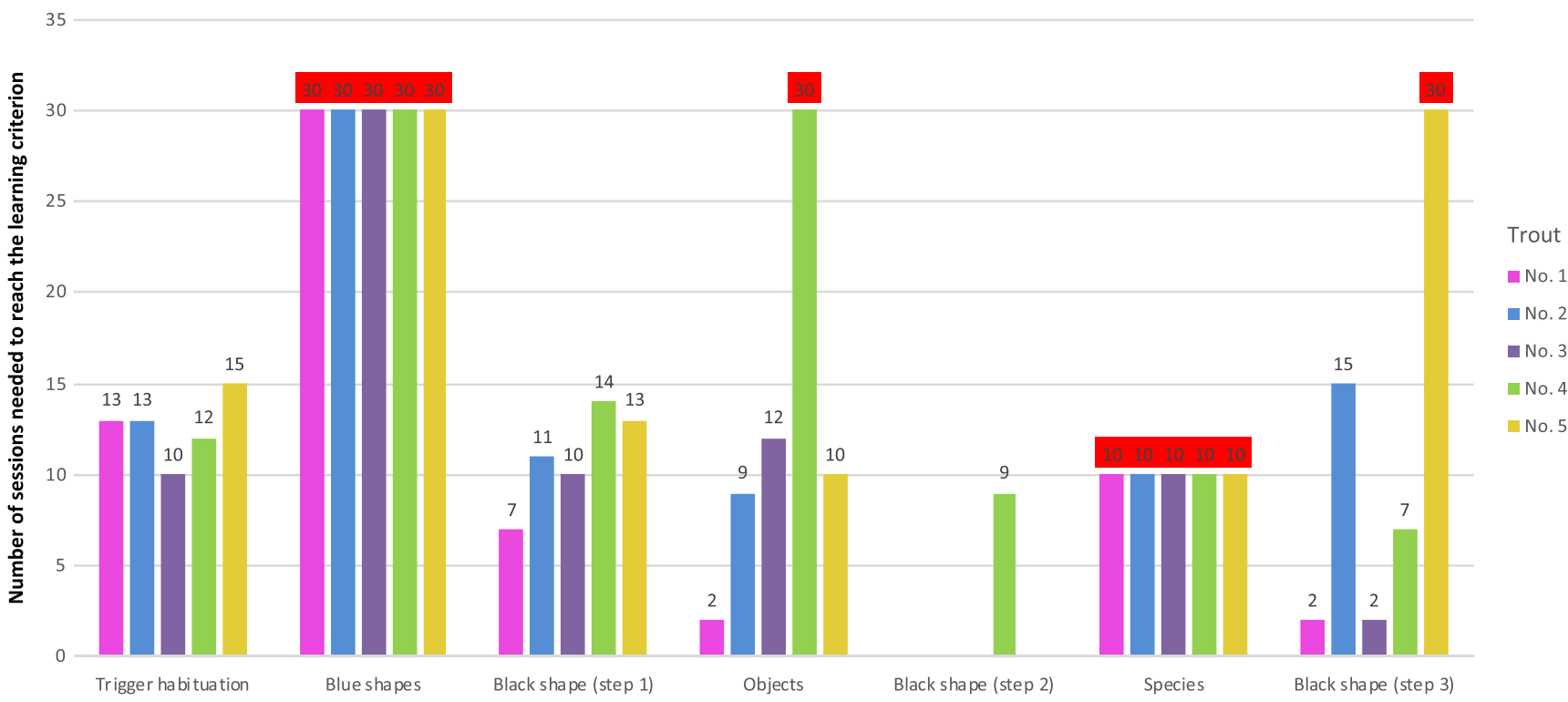

Fig. 3 Number of sessions needed to complete each stage by each individual (Nos. 1-5). The red labels indicate that the stage was not completed successfully

images of a black shape or any objects displayed on a screen but not between photographs of conspecifics and blue shapes or images of other fish species.

\section{Success and usefulness of the operant conditioning device}

In the habituation phase, fish were required to manipulate a trigger to obtain a food reward while being isolated in a novel tank. Nine individuals out of 15 completed this task and required between three and 15 sessions (ten trials each). This result coincides with that obtained for the African cichlid Pseudotropheus sp., which needed approximately $100 \pm 47$ trials (10 \pm 4.7 sessions) to get used to a 2-AFC device (approach the food holder to obtain a reward) (Schluessel et al., 2012). This performance is impressive in our case considering that the rainbow trout faced difficulties regarding the initial group-rearing conditions and ecology. Indeed, the first challenge might have been to become acclimatized alone in an unknown area, which represents a considerable source of stress for rainbow trout reared in groups. This situation resembles the novel-tank test commonly used to experimentally evaluate emotional reactivity of fish (Champagne, Hoefnagels, de Kloet, \& Richardson, 2010; Colson et al., 2015). Time was therefore necessary for acclimation and to associate netting and the novel tank with the positive event (highly appetitive

Table 2 Percentages of correct responses obtained by the five trout per session for each stage of the two-alternative forced-choice test task task

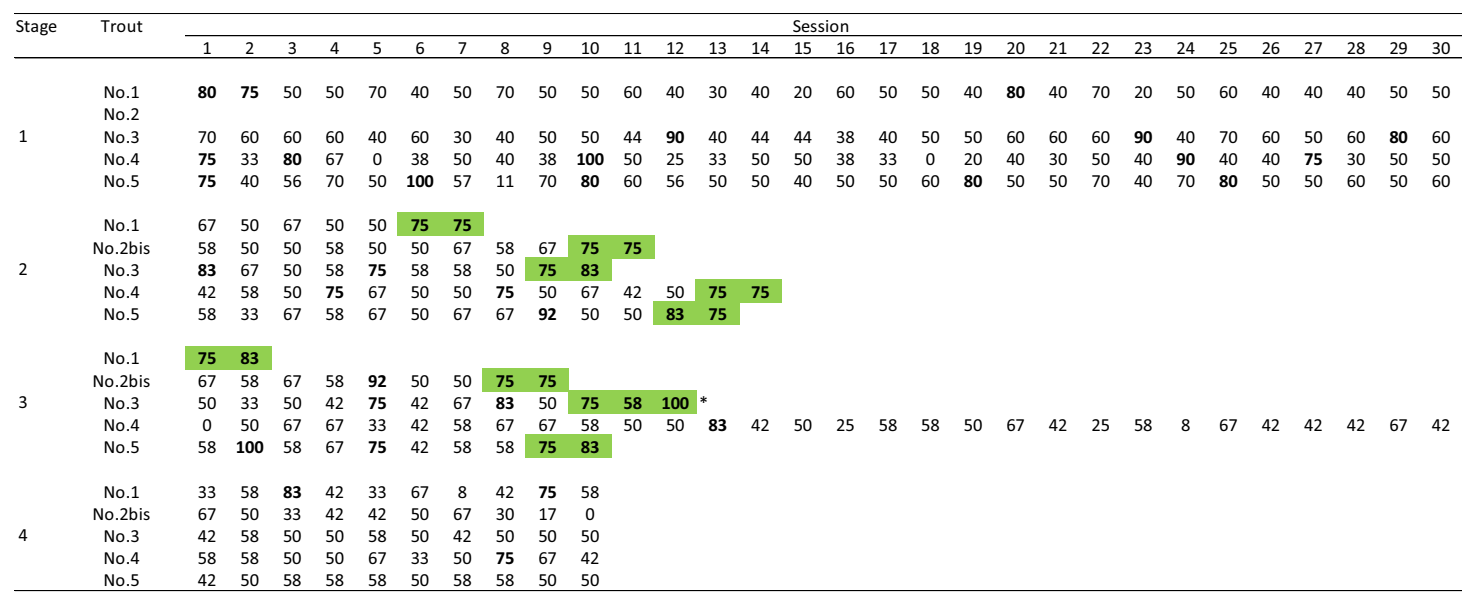

Percentages above $75 \%$ are bolded. The green highlighting indicates the two consecutive sessions in which an individual reached the learning criterion: $75 \%$ (or more) correct responses, which is significantly above chance threshold (50\%; binomial test: $\mathrm{P}=0.011, \mathrm{~N}=24$ trials).* Trout No. 3 reached the learning criterion in stage 3 because the three consecutive scores are significantly above chance threshold (50\%) when pooled (binomial test: $\mathrm{P}=0.0006$, $\mathrm{N}=36$ trials) 
food distribution). Then, the trout were required to learn how to activate a self-feeder individually outside of their initial context of group use. Trout reared in groups of 100-300 individuals require approximately 25 days to reach a stable level of selffeeding (Alanara \& Brannas, 1996). The trout therefore learned to handle a self-feeder trigger faster on their own - while being isolated in the test tank - than in a group since it took them $90 \pm$ 60 trials ( $9 \pm 6$ sessions). The use of an operant conditioning device with self-feeders positioned in front of corresponding visual stimuli represents in itself a significant performance because it involves a complete panel of cognitive abilities at the same time (spatial orientation, lever manipulation, object recognition, temporal and spatial association of environmental cues) (Jurado-Parras et al., 2013). To our knowledge, this is the first study demonstrating such cognitive performance in a farmed-fish species. Studies generally use discrete operant responses, such as approaching sensors (Siebeck et al., 2009), time spent near stimuli (Kuroda et al., 2017), passing through a door to enter a rewarded chamber (Bloch et al., 2019; GomezLaplaza et al, 2018; Mueller \& Neuhauss, 2012), but none where fish had to operate a lever as an active discrete conditioned response. With this last category of conditioned response, we demonstrated complex and complete cognitive abilities in rainbow trout. Two reasons may explain their rapid utilization of this operant conditioning device. First, trout were reared in enriched tanks (containing artificial plants, rocks, and pipes), environmental enrichment being known to enhance fish cognitive capacity (Salvanes et al., 2013; Strand et al., 2010).
However, the effect of environmental enrichment facilitating operant conditioning in fish deserves to be specifically tested in future studies. Secondly, the experimentation began when the trout were juveniles, a life stage with an important behavioral plasticity (Kotrschal \& Taborsky, 2010).

While the majority of fish studies requires the intervention of the experimenter between trials to correctly process the test (except in Brock, Sudwarts, Daggett, Parker, \& Brennan, 2017; Mueller \& Neuhauss, 2012), the present testing device was fully automated to prevent any fish disturbance. To measure behavior, automating procedures are commonly used in non-human primates and birds where subjects are required to make an active choice with touchscreen technologies and increase the reliability and standardization (within and between laboratory) (Brock et al., 2017). Our device brings for the first time in fish a reliable automated tool closer to the those used in other species, increasing both the flexibility and translational relevance of the results (Horner et al., 2013). This setup can be added to the existing repertoire of testing devices suitable for the investigation of simple (decision-making, object recognition) to more complex (working memory, numerical rules, categorization) cognitive abilities in fish.

\section{Forced-choice test: One stimulus}

The major challenge in the pretraining procedure was to create an association between a visual stimulus positioned behind one trigger (out of two) and the activation of the respective

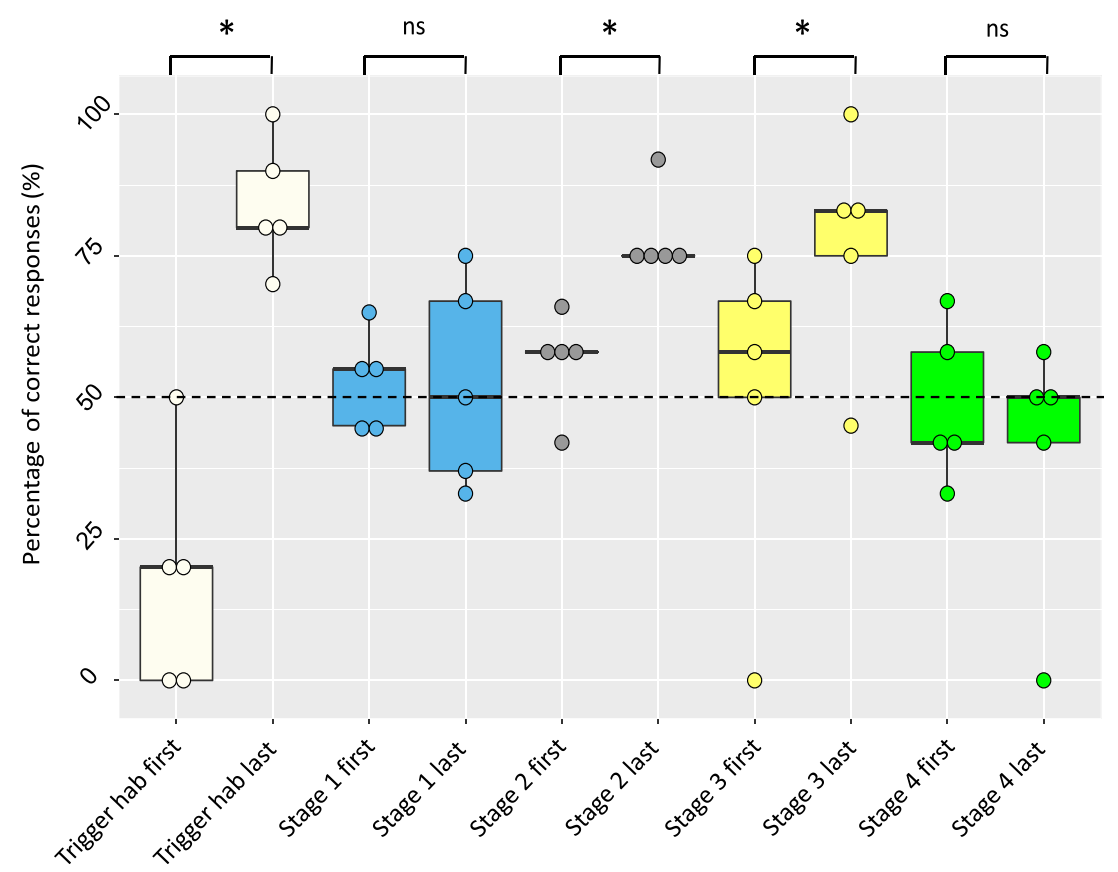

Fig. 4 Median (quartiles: 25\%, 75\%) percentages of correct responses during the first and last sessions for each stage: habituation to trigger activation, blue shapes as S- (stage 1), black shape as S- (stage 2, only step 1 is presented), objects as $\mathrm{S}$ - (stage 3 ) and other fish species as $\mathrm{S}$ (stage 4). A photograph of a trout is presented as $\mathrm{S}+$ in each two- alternative forced-choice test task test. Each color is dedicated to a stage, and dots represent the proportion of correct responses provided by the five individuals. The black dotted line represents chance level. Medians were compared between the first and last sessions using the Wilcoxon test (*: $\mathrm{P}<0.05 ;$ ns: nonsignificant; $\mathrm{N}=5$ ) 
trigger, which only then delivered food. Here, the rewarded stimulus was different shapes of different colors that changed between trials. After a total of 540 trials, no fish reached the learning criterion. Previous studies report that cichlids required $40 \pm 20$ trials (Schluessel et al., 2012), horses required between 840 and 1,100 trials (Gabor \& Gerken, 2012), and goats needed approximately 1,000 trials (Langbein et al., 2004) to learn a similar task but using only one or two different black symbols. The presentation of only one similar stimulus (or two) may have helped the fish to focus on the correct trigger and reach the learning criterion more easily in this task. However, a recent study showed that zebrafish were able to make a 2-AFC task by moving directly from the habituation stage to the 2-AFC paradigm without any presentation of a unique stimulus (Bloch et al., 2019). Therefore, pretraining using only one stimulus displayed on the screen may not have been necessary in our experimental design. Indeed, when the 2-AFC test began with a photograph of a conspecific as $\mathrm{S}+$ and a distracting stimulus (S-) displayed on either side, fish started to respond above chance level. As predicted by Fuss, Bleckmann, and Schluessel (2014), they seemed more attracted by a biologically relevant stimulus than to any kind of abstract shape. They also possibly needed the presence of a distracting stimulus, allowing the possibility to show a relative preference for the reinforced image of fish. Similarly, sharks need twice as many sessions to discriminate between a positive stimulus and a blank screen than between two distinct stimuli (Fuss et al., 2014). This is in accordance with Catania (1975), who demonstrated a preference for the availability of alternatives over unique stimuli in pigeons exposed to choice tests.

\section{Alternative forced-choice test: Two stimuli}

When the 2-AFC started, the distracting stimuli were represented by four blue shapes (and images of conspecifics as $\mathrm{S}+$ ), and no fish performed successfully within 30 sessions. Blue has been shown to be one of the preferred colors of rainbow trout (along with green) when given a choice (Luchiari \& Pirhonen, 2008; Maia et al., 2017). This visual preference for blue color may have caused a conflict of interest between the two stimuli presented on the screen, and affected the discrimination performance, as observed in zebrafish (Oliveira et al., 2015; Roy et al., 2019). Moreover, blue shapes were also presented as $\mathrm{S}+$ in the pretraining procedure and could have acted as a reversal instruction, increasing the conflict of interest between blue shapes as S- and images of conspecifics as S+. To increase the visual contrast between stimuli and because other species succeeded when using only one or two shapes as S- for a similar study design (Gabor \& Gerken, 2012; Langbein et al., 2004; Schluessel et al., 2012), a unique black shape was ultimately chosen as S- in stage 2 . This constituted a success since all the fish tested with this last combination met the learning criterion in a range of $126 \pm 42$ trials (10.5 \pm 3.5 sessions). Therefore, when given the appropriate stimuli, rainbow trout are able to learn a 2$\mathrm{AFC}$ task as quickly as higher-order vertebrates (horses: Gabor \& Gerken, 2012; goats: Langbein et al., 2004). Similar cognitive performance has also been demonstrated in other fish species. Cichlids took $116 \pm 90$ trials $(11.6 \pm 9$ sessions) and goldfish took 2,000-3,500 trials (20-35 sessions) to discriminate between a square and a triangle (Schluessel et al., 2012). The gray bamboo shark needed 98 \pm 50 trials (9.75 \pm 5.04 sessions) (Fuss et al., 2014) to reach a $75 \%$ correct response level in the same task (discriminating a square from a triangle). In a 2-AFC test based on color discrimination (red and green), five sessions were sufficient for zebrafish to meet the learning criterion (Bloch et al., 2019). Here, we show for the first time similar discriminative abilities between two different visual stimuli (a black star and photographs of conspecifics) in a selected farmed fish species while other studies used principally wild fish or fish from pet stores.

The fish decision strategy could have been to avoid S- on the basis of its characteristics instead of associating the photograph of a conspecific with a reward, as previously observed in Peters' elephant-nose fish (von der Emde \& Fetz, 2007). However, four individuals (out of five) were successful in discriminating between images of conspecifics and objects in $7 \pm 5$ sessions in stage 3 . This indicates that the fish did not simply learn to avoid the black star in the previous stage but rather chose the $\mathrm{S}+$ because of its positive association with the reward. We cannot exclude that an innate visual preference for photographs of conspecifics has explained this result. These preferences can be very strong and affect discrimination performance, as shown in other fish species (Oliveira et al., 2015; Roy et al., 2019). However, the fact that performance significantly changed between the first and the last sessions suggests the role of learning here. Also, trout failed to reach the learning criterion in stage 1 and stage 4 , which suggests that they have no innate preference for the photographs of conspecifics presented as $\mathrm{S}+$. To validate this finding, it would be interesting to use the photographs used here as $\mathrm{S}+$ as the $\mathrm{S}$ in a future study. The individual that did not succeed in this task was tested again in stage 2 to control for its overall performance in the 2-AFC task, and it discriminated again between the black star and the images of conspecifics in nine sessions. This confirms that this individual did not stop being motivated to use the apparatus but was unable to perform correctly in stage 3 , illustrating the intraspecific variability in behavioral responses existing in rainbow trout. We noticed that this individual was easily stressed, and any perturbation (here, a novel stage) was sufficient to induce behavioral inhibition, resulting in the stress-coping style described in highly responsive strains of rainbow trout (Overli et al., 2007). The 60 objects used as S- in stage 3 were all taken from the internet 
and were as various as a clock, an armchair, a computer, a desk, and a bottle. No object was particularly preferred/more often chosen by the fish during all the sessions, and the only common feature among them was their nonbiological relevance for fish. Schluessel et al. (2012) showed that the African cichlid Pseudotropheus sp. distinguished between two mental categories ("fish" and "snail") by examining the entire stimulus rather than individual features. The authors consider that since predators, prey, conspecifics, and objects (shelters or landmarks) present in a habitat may vary in size, shape, and color, using general similar features provides fish with a certain degree of flexibility to react appropriately. Here, fish might have distinctly memorized or classified the two categories "objects" and "fish" in their entirety as nonbiologically relevant and biologically relevant, or non-fish and fish, respectively.

When discrimination difficulty increased in stage 4, where photographs of other fish species were displayed as S- (and conspecifics as $\mathrm{S}+$ ), performances dropped to the chance threshold. In this case, the visual difference between $\mathrm{S}$ - and $\mathrm{S}+$ was not as obvious as that between a fish and an object, and all individuals failed in the two-discrimination task. However, we know that teleost fish are able to see complex and contrasting patterns (Ben-Simon et al., 2012; Champ et al., 2014; Gierszewski et al., 2013), but note that none of these studies have specifically focused on the visual acuity of rainbow trout. Rainbow trout recognize visually their conspecifics (Höjesjö et al., 2015; Johnsson \& Åkerman, 1998), but no study investigated whether rainbow trout can discriminate them from heterospecifics. Here, we considered that the fish recognized the general outline of a conspecific as a fish but similarly recognized the shape of any other fish species. If trout learned the category "fish" (all species combined because of biological relevance) rather than remembering the four photographs of their conspecifics, this could explain the poor performance observed in stage 4 , linked to the shared features between the positive and the negative stimulus. However, three scores reached the $75 \%$ level of correct responses but in nonconsecutive sessions. A maximum of ten sessions was chosen for this stage since the instruction given (discriminating between two images of fish) was considered to be too ambiguous and more than ten sessions would have perhaps cancelled the initial positive association of an image of a trout with a reward and could have acted as a reversal learning, as shown in zebrafish (Kuroda et al., 2017). Therefore, perhaps a few more sessions would have been necessary to know with accuracy whether rainbow trout are able to visually distinguish between conspecifics and other fish species in this experimental set up. An extra experiment running stage 4 for more than ten sessions is necessary and is currently planned to elucidate this question. Another explanation for this failure in stage 4 concerns the use of static images displayed on screen monitors in the investigation of live animal discrimination. Many fish species - including rainbow trout (Höjesjö et al., 2015; Johnsson \& Åkerman, 1998) - are able to recognize live conspecifics individually using visual cues (Culumber, 2015; Griffiths \& Magurran, 1999; Roux et al., 2016). However, when males and females of the peacock blenny (Salaria pavo) were presented with a live conspecific male or a video of the same individual, the video images failed to elicit appropriate behavioral responses (Goncalves, Oliveira, Korner, Poschadel, \& Schlupp, 2000). In the case of anemonefishes (Johnston \& Dixson, 2017) and Lake Malawi cichlid species (Knight \& Turner, 1999), it was shown that a combination of visual and other senses stimuli (chemical cues) can be fundamental in conspecific recognition. The importance of olfactory versus visual cues in conand hetero-specifics recognition varies across species (Santacà, Busatta, Lucon-Xiccato \& Bisazza, 2019). As in other fish species (coral reef damselfish: Holmes \& McCormick, 2010; zebrafish: Speedie \& Gerlai, 2008; cichlids: Vavrek \& Brown, 2009), rainbow trout can perceive conspecifics using chemical cues (Brown \& Smith, 1997). In the motionless 2-D images displayed on the screen, rainbow trout could perceive neither behavioral features nor other sensory cues (olfaction or mechanoreception) present in live fish. In this way, 2-D images may not be appropriate for rainbow trout to recognize between con- and hetero-specifics.

Interestingly, an effect of trout's running order was observed only at this stage. An individual tested at the last place (fifth) presented weaker performances than when tested earlier. One could have predicted the opposite result, fish being tested earlier in the day (closer to their last feeding) being less motivated to receive the food-reward than others. However, the overnight food-deprivation period varied between fish from $18 \mathrm{~h}$ to $24 \mathrm{~h}$ (depending on their running order), which is a duration long enough for all of them to ensure a similar food motivation. More importantly, when a stage appeared easier to the fish (stages 2 and 3), running order did not affect the conditioned responses. The time of passage possibly interfered with the stress accumulated by repeated netting in the rearing tank, affecting the score of the last fish netted at stage 4 only, when the instruction given required all fish attention. Indeed, acute stress is known to impair fish attention and learning performance (Gaikwad et al., 2011). To control for their overall performance in the 2-AFC task, the black star was again presented as S-, and four individuals (out of five) regained the criterion level in stage 2-step 3. Again, the failure to discriminate between two images of fish (con- and heterospecifics) was thus not simply due to a lack of motivation to answer.

In training procedures, the success of a few individuals is sufficient to generalize proven cognitive abilities to the whole species. In some cases, even one individual completing a task is adequate, meaning that the brain of the members of the species is endowed with neurocognitive systems that likely 
support the resolution of the task (Agrillo \& Bisazza, 2014). As in the present experiment, animal cognitive studies generally include few experimental subjects (archerfish: six individuals tested: Newport, Wallis, \& Siebeck, 2015; sheep: eight individuals tested: Knolle et al., 2017; horses: seven individuals tested: Gabor \& Gerken, 2012). We can therefore consider that in our experimental paradigm, rainbow trout species are capable of discriminating in a 2-AFC task between a photograph of conspecifics and a black star (five individuals succeeding) and between conspecifics and any other object (four individuals succeeding). This finding adds to the body of evidence that fish have good learning skills, even in the absence of a neocortex, the cerebral region involved in learning processes in mammals (Seger \& Miller, 2010), and presents a novel method with which to study a diverse range of fish discrimination abilities.

\section{Rainbow trout cognitive performance under farming conditions}

The present study demonstrates that a farmed fish species is capable of object recognition and likely possesses categorization learning abilities. Distinguishing items in the environment - i.e., conspecifics and objects (pipes or landmarks) present in the rearing tank - in an organized manner (categories) allows fish to solve problems, communicate in groups, and obtain information (Oliveira et al., 2015). Their ability to visually recognize different items present in the environment and to memorize them might reduce costly efforts to consider as a new information the same item when encountered again. Individual recognition may even help to decrease aggression by ensuring a stable social hierarchy since trout avoid repeating costly social interactions when they have already confronted each other's (Johnsson, 1997; Johnsson \& Akerman, 1998). Finally, distinguishing those items without ambiguity certainly allows rainbow trout to spend time on other essential activities (foraging, exploring) and reduce stress due to the inherent unpredictability of captive environments.

The performance exhibited by rainbow trout while using the conditioned device opens new perspectives concerning the possible cognitive enrichments to be developed in future farming systems. Cognitive enrichment, also known as occupational or psychological enrichment, represents the possibility for animals to meet moderate challenges by using their cognitive abilities and/or to actively interact with their environment (Manteuffel et al., 2009; Meehan \& Mench, 2007; Oesterwind et al., 2016). Challenges range from simple manipulations (devices to obtain food) to more complex challenges (puzzle solving) tailored to the ecology of the species. It mainly aims at reducing psychological monotony of oftenimpoverished environments by introducing predictable and/or animal-controllable variations (Naslund \& Johnsson, 2016).
Reducing the psychological monotony of impoverished environments might become more important after highlighting the complex cognitive performance displayed by rainbow trout through this experiment. This knowledge could be applied to provide stimulations for engaging their advanced cognitive capacities, in order to improve their rearing conditions. For instance, novel cognitive enrichments could include visual stimulations inside the tanks or "improved" self-feeders (delivering food only when a goal is achieved), and would enhance fish welfare under future farming conditions.

These findings are also important in a welfare context since evidence that farm animals have cognitive skills comparable to those of so-called higher vertebrates encourages social awareness of the need to improve their welfare.

\section{Conclusions}

To conclude, rainbow trout rapidly learned how to operate a self-feeder individually in conditions of social isolation. This reliable automated tool closer to the operant setup used in higher species can be added to the existing repertoire of testing devices suitable for the investigation of cognitive abilities in fish. We also showed that rainbow trout are able to discriminate a pool of four recurrent conspecifics from a unique black shape and from different objects but not from other fish species. Whether the trout needed more than ten trials to demonstrate their ability to discriminate visually between con- and hetero-specifics, if they learned a category "fish shape" or "biologically relevant" and thus could not distinguish between the positive and negative stimuli presented because of shared features, or if they instead needed to see the two "fish" stimuli live to perceive any behavioral or other sensory features, still needs to be further tested in dedicated experiments. With the visual items chosen for the 2-AFC task, we cannot completely exclude that at some of the results were due to innate visual preferences for the photographs of conspecifics, but the fact that the performance increased between the first and the last session suggests the role of learning here. The ability to visually recognize different items present in the environment and to memorize them reduces costly efforts to consider as new information the same item when encountered again.

This study sheds new light on the mental capacities of rainbow trout, a farmed animal often forgotten in public concerns. Its success in operating self-feeders in a complex situation may help us to develop species-specific ethologicalbased tools, or cognitive enrichments, in order to increase the welfare of the fish in future farming systems.

Acknowledgements This experiment was made possible through funding provided by the Department PHASE (Animal Physiology and Breeding Systems) of INRAE. 


\section{References}

Agrillo, C., Piffer, L., \& Bisazza, A. (2010). Large number discrimination by mosquitofish. PLoS One, 5(12), e15232. https://doi.org/10.1371/ journal.pone.0015232.

Agrillo, C., \& Bisazza, A. (2014). Spontaneous versus trained numerical abilities. A comparison between the two main tools to study numerical competence in non-human animals. Journal of Neuroscience Methods, 234, 82-91. https://doi.org/10.1016/j.jneumeth.2014.04. 027.

Alanara, A., \& Brannas, E. (1996). Dominance in demand-feeding behaviour in arctic charr and rainbow trout: the effect of stocking density. Journal of Fish Biology, 48(2), 242-254. https://doi.org/ 10.1111/j.1095-8649.1996.tb01116.x.

Ben-Simon, A., Ben-Shahar, O., Vasserman, G., Ben-Tov, M., \& Segev, R. (2012). Visual acuity in the archerfish: behavior, anatomy, and neurophysiology. Journal of Vision, 12(12), 18. https://doi.org/10. $1167 / 12.12 .18$

Bloch, S., Froc, C., Pontiggia, A., \& Yamamoto, K. (2019). Existence of working memory in teleosts: establishment of the delayed matchingto-sample task in adult zebrafish. Behavioural Brain Research, 370, 111924. https://doi.org/10.1016/j.bbr.2019.111924.

Brock, A. J., Sudwarts, A., Daggett, J., Parker, M. O., \& Brennan, C. H. (2017). A fully automated computer-based 'Skinner Box' for testing learning and memory in zebrafish. BioRxiv, 110478. https://doi.org/ $10.1101 / 110478$.

Broglio, C., Rodriguez, F., \& Salas, C. (2003). Spatial cognition and its neural basis in teleost fishes. Fish and Fisheries, 4(3), 247-255. https://doi.org/10.1046/j.1467-2979.2003.00128.x.

Brown, G. E., \& Smith, R. J. F. (1997). conspecific skin extracts elicit antipredator responses in juvenile rainbow trout (oncorhynchus mykiss). Canadian Journal of Zoology-Revue Canadienne De Zoologie, 75(11), 1916-1922. https://doi.org/10.1139/z97-821.

Bshary, R., \& Grutter, A. S. (2006). Image scoring and cooperation in a cleaner fish mutualism. Nature, 441(7096), 975-978. https://doi. org/10.1038/nature04755.

Bshary, R., Wickler, W., \& Fricke, H. (2002). Fish cognition: a primate's eye view. Animal Cognition, 5(1), 1-13. https://doi.org/10.1007/ s10071-001-0116-5.

Cañon Jones, H. A., Noble, C., Damsgård, B., \& Pearce, G. P. (2012). Investigating the influence of predictable and unpredictable feed delivery schedules upon the behaviour and welfare of Atlantic salmon parr (Salmo salar) using social network analysis and fin damage. Applied Animal Behaviour Science, 138(1-2), 132-140. https://doi. org/10.1016/j.applanim.2012.01.019.

Catania, A. C. (1975). Pigeons preference for free choice over forced choice as a function of number of free-choice alternatives. Bulletin of the Psychonomic Society, 6(4), 424-424.

Champ, C., Wallis, G., Vorobyev, M., Siebeck, U., \& Marshall, J. (2014). Visual acuity in a species of coral reef fish: rhinecanthus aculeatus. Brain Behavior and Evolution, 83(1), 31-42. https://doi.org/10. $1159 / 000356977$

Champagne, D. L., Hoefnagels, C. C. M., de Kloet, R. E., \& Richardson, M. K. (2010). Translating rodent behavioral repertoire to zebrafish (danio rerio): relevance for stress research. Behavioural Brain Research, 214(2), 332-342. https://doi.org/10.1016/j.bbr.2010.06. 001

Clark, D. L., \& Stephenson, K. R. (1999). Response to video and computer-animated images by the tiger barb, puntius tetrazona. Environmental Biology of Fishes, 56(3), 317-324. https://doi.org/ 10.1023/A:1007549721631.

Colson, V., Cousture, M., Damasceno, D., Valotaire, C., Nguyen, T., Le Cam, A., \& Bobe, J. (2019). Maternal temperature exposure impairs emotional and cognitive responses and triggers dysregulation of neurodevelopment genes in fish. PeerJ, 7, e6338. https://doi.org/ 10.7717/peerj.6338

Colson, V., Sadoul, B., Valotaire, C., Prunet, P., Gaumé, M., \& Labbé, L. (2015). Welfare assessment of rainbow trout reared in a recirculating aquaculture system: comparison with a flow-through system. Aquaculture, 436(0), 151-159. https://doi.org/10.1016/j. aquaculture.2014.10.047.

Culumber, Z. W. (2015). Early recognition and response to predator, heterospecific, and conspecific visual cues by multiple species of poeciliid fry. Behaviour, 152(11), 1463-1479. https://doi.org/10. 1163/1568539x-00003287.

DeLong, C. M., Barbato, S., O'Leary, T., \& Wilcox, K. T. (2017). Small and large number discrimination in goldfish (Carassius auratus) with extensive training. Behavioural Processes, 141, 172-183. https:// doi.org/10.1016/j.beproc.2016.11.011.

FEAP (2017). FEAP Annual Report 2017. https://issuu.com/feapsec/ docs/feap_ar2017.

Fuss, T., Bleckmann, H., \& Schluessel, V. (2014). Visual discrimination abilities in the gray bamboo shark (chiloscyllium griseum). Zoology (Jena), 117(2), 104-111. https://doi.org/10.1016/j.zool.2013.10. 009.

Franks, B. (2018). Cognition as a cause, consequence, and component of welfare. In Advances in agricultural animal welfare: science and practice (p. 22). https://doi.org/10.1016/B978-0-08-101215-4. 00001-8.

Gabor, V., \& Gerken, M. (2012). Cognitive testing in horses using a computer based apparatus. Applied Animal Behaviour Science, 139(3-4), 242-250. https://doi.org/10.1016/j.applanim.2012.04. 010.

Gaikwad, S., Stewart, A., Hart, P., Wong, K., Piet, V., Cachat, J., \& Kalueff, A. V. (2011). Acute stress disrupts performance of zebrafish in the cued and spatial memory tests: the utility of fish models to study stress-memory interplay. Behavioural Processes, 87(2), 224-230. https://doi.org/10.1016/j.beproc.2011.04.004.

Gerullis, P., \& Schuster, S. (2014). Archerfish actively control the hydrodynamics of their jets. Current Biology, 24(18), 2156-2160. https:// doi.org/10.1016/j.cub.2014.07.059.

Gierszewski, S., Bleckmann, H., \& Schluessel, V. (2013). Cognitive abilities in malawi cichlids (Pseudotropheus sp.): matching-to-sample and image/mirror-image discriminations. PLoS One, 8(2), e57363. https://doi.org/10.1371/journal.pone.0057363.

Goldman, M., \& Shapiro, S. (1979). Matching-to-sample and oddityfrom-sample in goldfish. Journal of the Experimental Analysis of Behavior, 31(2), 259-266. https://doi.org/10.1901/jeab.1979.31259.

Gómez-Laplaza, L. M., Díaz-Sotelo, E., \& Gerlai, R. (2018). Quantity discrimination in angelfish, Pterophyllum scalare: A novel approach with food as the discriminant. Animal Behaviour, 142, 19-30. https://doi.org/10.1016/j.anbehav.2018.06.001.

Gómez-Laplaza, L. M., \& Gerlai, R. (2013). The role of body surface area in quantity discrimination in angelfish (pterophyllum scalare). PLoS One, 8(12), e83880. https://doi.org/10.1371/journal.pone.0083880.

Goncalves, D. M., Oliveira, R. F., Korner, K., Poschadel, J. R., \& Schlupp, I. (2000). Using video playbacks to study visual communication in a marine fish, salaria pavo. Animal Behaviour, 60, 351357. https://doi.org/10.1006/anbe.2000.1459.

Griffiths, S. W., \& Magurran, A. E. (1999). Schooling decisions in gunnies (poecilia reticulata) are based on familiarity rather than kin recognition by phenotype matching. Behavioral Ecology and Sociobiology, 45(6), 437-443. https://doi.org/10.1007/ s002650050582.

Grosenick, L., Clement, T. S., \& Fernald, R. D. (2007). Fish can infer social rank by observation alone. Nature, 445(7126), 429-432. https://doi.org/10.1038/nature05511.

Herman, L. M., Gory, J. D., Hovancik, J. R., \& Bradshaw, G. L. (1989). Generalization of visual matching by a bottlenosed dolphin 
(tursiops-truncatus) - evidence for invariance of cognitive performance with visual and auditory materials. Journal of Experimental Psychology-Animal Behavior Processes, 15(2), 124-136. https:// doi.org/10.1037/0097-7403.15.2.124.

Hester, F. J. (1968). Visual contrast thresholds of the goldfish (Carassius auratus). Vision Research, 8(10), 1315-1336.

Höjesjö, J., Axelsson, M., Dahy, R., Gustavsson, L., \& Johnsson, J. I. (2015). Sight or smell? Behavioural and heart rate responses in subordinate rainbow trout exposed to cues from dominant fish. PeerJ, 3. https://doi.org/10.7717/peerj.1169.

Holmes, T. H., \& McCormick, M. I. (2010). Smell, learn and live: the role of chemical alarm cues in predator learning during early life history in a marine fish. Behavioural Processes, 83(3), 299-305. https://doi. org/10.1016/j.beproc.2010.01.013.

Horner, A. E., Heath, C. J., Hvoslef-Eide, M., Kent, B. A., Kim, C. H., Nilsson, S. R. O., et al. (2013). The touchscreen operant platform for testing learning and memory in rats and mice. Nature Protocols, 8(10), 1961-1984. https://doi.org/10.1038/nprot.2013.122.

Ingraham, E., Anderson, N. D., Hurd, P. L., \& Hamilton, T. J. (2016). Twelve-day reinforcement-based memory retention in african cichlids (labidochromis caeruleus). Frontiers in Behavioral Neuroscience, 10, 157. https://doi.org/10.3389/fnbeh.2016.00157.

Ioannou, C. C., Couzin, I. D., James, R., Croft, D. P., \& Krause, J. (2011). Social organization and information transfer in schooling fish. In C. Brown, K. Laland, \& J. Krause (Eds.), Fish cognition and behaviour (2nd ed.). Oxford: Wiley-Blackwell. https://doi.org/10.1002/ 9781444342536.ch10.

Johnsson, J. I. (1997). Individual recognition affects aggression and dominance relations in rainbow trout, oncorhynchus mykiss. Ethology, 103(4), 267-282. https://doi.org/10.1111/j.1439-0310.1997. tb00017.x.

Johnsson, J. I., \& Åkerman, A. (1998). Watch and learn : Preview of the fighting ability of opponents alters contest behaviour in rainbow trout. Animal Behaviour, 56(3), 771-776. https://doi.org/10.1006/ anbe.1998.0824.

Johnston, N. K., \& Dixson, D. L. (2017). Anemonefishes rely on visual and chemical cues to correctly identify conspecifics. Coral Reefs, 36, 903-912. https://doi.org/10.1007/s00338-017-1582-9.

Jurado-Parras, M. T., Sanchez-Campusano, R., Castellanos, N. P., PdelPozo, F., Gruart, A., \& Delgado-Garcia, J. M. (2013). Differential contribution of hippocampal circuits to appetitive and consummatory behaviors during operant conditioning of behaving mice. Journal of Neuroscience, 33(6), 2293-2304. https://doi.org/10.1523/ JNEUROSCI.1013-12.2013.

Knight, M. E., \& Turner, G. F. (1999). Reproductive isolation among closely related Lake Malawi cichlids : Can males recognize conspecific females by visual cues? Animal Behaviour, 58(4), 761-768. https://doi.org/10.1006/anbe.1999.1206.

Knolle, F., Goncalves, R. P., \& Morton, A. J. (2017). Sheep recognize familiar and unfamiliar human faces from two-dimensional images. Royal Society Open Science, 4(11), 171228. https://doi.org/10.1098/ rsos.171228.

Kotrschal, A., \& Taborsky, B. (2010). Environmental change enhances cognitive abilities in fish. PLoS Biology, 8(4), e1000351. https://doi. org/10.1371/journal.pbio.1000351.

Kuroda, T., Mizutani, Y., Cancado, C. R. X., \& Podlesnik, C. A. (2017). Reversal learning and resurgence of operant behavior in zebrafish (danio rerio). Behavioural Processes, 142, 79-83. https://doi.org/10. 1016/j.beproc.2017.06.004.

Langbein, J., Nurnberg, G., \& Manteuffel, G. (2004). Visual discrimination learning in dwarf goats and associated changes in heart rate and heart rate variability. Physiology \& Behavior, 82(4), 601-609. https://doi.org/10.1016/j.physbeh.2004.05.007.

Luchiari, A. C., \& Pirhonen, J. (2008). Effects of ambient colour on colour preference and growth of juvenile rainbow trout oncorhynchus mykiss (walbaum). Journal of Fish Biology, 72(6), 1504-1514. https://doi.org/10.1111/j.1095-8649.2008.01824.x.

Lucon-Xiccato, T., \& Bisazza, A. (2014). Discrimination reversal learning reveals greater female behavioural flexibility in guppies. Biology Letters, 10(6), 20140206. https://doi.org/10.1098/rsbl.2014.0206.

Maia, C. M., Ferguson, B., Volpato, G. L., \& Braithwaite, V. A. (2017). Physical and psychological motivation tests of individual preferences in rainbow trout. Journal of Zoology, 302(2), 108-118. https://doi.org/10.1111/jzo.12438.

Manteuffel, G., Langbein, J., \& Puppe, B. (2009). From operant learning to cognitive enrichment in farm animal housing: bases and applicability. Animal Welfare, 18(1), 87-95.

Martins, C. I. M., Galhardo, L., Noble, C., Damsgard, B., Spedicato, M. T., Zupa, W., Beauchaud, M., Kulczykowska, E., Massabuau, J. C., Carter, T., Planellas, S. R., \& Kristiansen, T. (2012). Behavioural indicators of welfare in farmed fish. Fish Physiology and Biochemistry, 38(1), 17-41. https://doi.org/10.1007/s10695-0119518-8.

Meehan, C. L., \& Mench, J. A. (2007). The challenge of challenge: Can problem solving opportunities enhance animal welfare? Applied Animal Behaviour Science, 102(3-4), 246-261. https://doi.org/10. 1016/j.applanim.2006.05.031.

Mueller, K., \& Neuhauss, S. (2012). Automated visual choice discrimination learning in zebrafish (Danio rerio). Journal of Integrative Neuroscience, 11, 73-85. https://doi.org/10.1142/ S0219635212500057.

Näslund, J., \& Johnsson, J. I. (2016). Environmental enrichment for fish in captive environments: effects of physical structures and substrates. Fish and Fisheries, 17(1), 1-30. https://doi.org/10.1111/ faf. 12088 .

Neumeyer, C. (2003). Wavelength dependence of visual acuity in goldfish. Journal of Comparative Physiology A: Sensory, Neural, and Behavioral Physiology, 189(11), 811-821. https://doi.org/10.1007/ s00359-003-0457-4.

Newport, C., Wallis, G., \& Siebeck, U. E. (2015). Same/different abstract concept learning by archerfish (toxotes chatareus). PLoS One, 10(11), e0143401. https://doi.org/10.1371/journal.pone.0143401.

Newport, C., Wallis, G., Temple, S. E., \& Siebeck, U. E. (2013). Complex, context-dependent decision strategies of archerfish, toxotes chatareus. Animal Behaviour, 86(6), 1265-1274. https:// doi.org/10.1016/j.anbehav.2013.09.031.

Oesterwind, S., Nürnberg, G., Puppe, B., \& Langbein, J. (2016). Impact of structural and cognitive enrichment on the learning performance, behavior and physiology of dwarf goats (Capra aegagrus hircus). Applied Animal Behaviour Science, 177, 34-41. https://doi.org/10. 1016/j.applanim.2016.01.006.

Oliveira, J., Silveira, M., Chacon, D., \& Luchiari, A. (2015). The zebrafish world of colors and shapes: preference and discrimination. Zebrafish, 12(2), 166-173. https://doi.org/10.1089/zeb.2014.1019.

Overli, O., Sorensen, C., Pulman, K. G., Pottinger, T. G., Korzan, W., Summers, C. H., \& Nilsson, G. E. (2007). Evolutionary background for stress-coping styles: relationships between physiological, behavioral, and cognitive traits in non-mammalian vertebrates. Neuroscience \& Biobehavioral Reviews, 31(3), 396-412. https:// doi.org/10.1016/j.neubiorev.2006.10.006.

Parker, M. O., Gaviria, J., Haigh, A., Millington, M. E., Brown, V. J., Combe, F. J., \& Brennan, C. H. (2012). Discrimination reversal and attentional sets in zebrafish (danio rerio). Behavioural Brain Research, 232(1), 264-268. https://doi.org/10.1016/j.bbr.2012.04. 035.

Paśko, Ł. (2010). Tool-like behavior in the sixbar wrasse, Thalassoma hardwicke (Bennett, 1830). Zoo Biology, 29(6), 767-773. https:// doi.org/10.1002/zoo.20307.

Rodriguez, F., Duran, E., Vargas, J. P., Torres, B., \& Salas, C. (1994). Performance of goldfish trained in allocentric and egocentric maze procedures suggests the presence of a cognitive mapping system in 
fishes. Animal Learning \& Behavior, 22(4), 409-420. https://doi. org/10.3758/bf03209160.

Roux, N., Duran, E., Lanyon, R. G., Frederich, B., Berthe, C., Besson, M., Dixson, D. L., \& Lecchini, D. (2016). Brain lateralization involved in visual recognition of conspecifics in coral reef fish at recruitment. Animal Behaviour, 117, 3-8. https://doi.org/10.1016/j. anbehav.2016.04.011.

Roy, T., Suriyampola, P. S., Flores, J., López, M., Hickey, C., Bhat, A., \& Martins, E. P. (2019). Color preferences affect learning in zebrafish, Danio rerio. Scientific Reports, 9, 14531. https://doi.org/10.1038/ s41598-019-51145-5.

Santacà, M., Busatta, M., Lucon-Xiccato, T., \& Bisazza, A. (2019). Sensory differences mediate species variation in detour task performance. Animal Behaviour, 155, 153-162. https://doi.org/10.1016/j. anbehav.2019.05.022.

Satoh, S., Tanaka, H., \& Kohda, M. (2016). Facial recognition in a discus fish (cichlidae): experimental approach using digital models. PLoS One, 11(5). https://doi.org/10.1371/journal.pone.0154543.

Salvanes, A. G., Moberg, O., Ebbesson, L. O., Nilsen, T. O., Jensen, K. H., \& Braithwaite, V. A. (2013). Environmental enrichment promotes neural plasticity and cognitive ability in fish. Proceedings Biological Sciences, 280(1767), 20131331. https://doi.org/10. 1098/rspb.2013.1331.

Schluessel, V., Fricke, G., \& Bleckmann, H. (2012). Visual discrimination and object categorization in the cichlid Pseudotropheus sp. Animal Cognition, 15(4), 525-537. https://doi.org/10.1007/ s10071-012-0480-3.

Seger, C. A., \& Miller, E. K. (2010). Category learning in the brain. Annual Review of Neuroscience, 33, 203-219. https://doi.org/10. 1146/annurev.neuro

Shettleworth, S. J. (2009). Cognition, evolution, and behavior. Oxford University Press.

Sidman, M., Rauzin, R., Lazar, R., Cunningham, S., Tailby, W., \& Carrigan, P. (1982). A Search for symmetry in the conditional discriminations of rhesus monkeys, baboons, and children. Journal of the Experimental Analysis of Behavior, 37(1), 23-44. https://doi. org/10.1901/jeab.1982.37-23.

Siebeck, U. E., Litherland, L., \& Wallis, G. M. (2009). Shape learning and discrimination in reef fish. Journal of Experimental Biology, 212(13), 2113-2119. https://doi.org/10.1242/jeb.028936.
Sovrano, V. A., \& Bisazza, A. (2008). Recognition of partly occluded objects by fish. Animal Cognition, 11(1), 161-166. https://doi.org/ 10.1007/s10071-007-0100-9.

Speedie, N., \& Gerlai, R. (2008). Alarm substance induced behavioral responses in zebrafish (danio rerio). Behavioural Brain Research, 188(1), 168-177. https://doi.org/10.1016/j.bbr.2007.10.031.

Strand, D. A., Utne-Palm, A. C., Jakobsen, P. J., Braithwaite, V. A., Jensen, K. H., \& Salvanes, A. G. V. (2010). Enrichment promotes learning in fish. Marine Ecology Progress Series, 412, 273-282. https://doi.org/10.3354/meps08682.

Vavrek, M. A., \& Brown, G. E. (2009). Threat-sensitive responses to disturbance cues in juvenile convict cichlids and rainbow trout Annales Zoologici Fennici, 46(3), 171-180. https://doi.org/10. 5735/086.046.0302.

von der Emde, G., \& Fetz, S. (2007). Distance, shape and more: recognition of object features during active electrolocation in a weakly electric fish. Journal of Experimental Biology, 210(17), 3082-3095. https://doi.org/10.1242/jeb.005694.

Vonk, J. (2003). Gorilla (gorilla gorilla gorilla) and orangutan (pongo abelii) understanding of first- and second-order relations. Animal Cognition, 6(2), 77-86. https://doi.org/10.1007/s10071-003-0159$\mathrm{x}$.

Wyzisk, K., \& Neumeyer, C. (2007). Perception of illusory surfaces and contours in goldfish. Visual Neuroscience, 24, 291-298. https://doi. org/10.1017/S095252380707023X.

Zerbolio, D. J., \& Royalty, J. L. (1983). Matching and oddity conditional discrimination in the goldfish as avoidance responses: evidence for conceptual avoidance learning. Animal Learning \& Behavior, 11(3), 341-348. https://doi.org/10.3758/bf03199786.

Open Practices Statements The data and materials for all experiments will be available on publication.

Publisher's note Springer Nature remains neutral with regard to jurisdictional claims in published maps and institutional affiliations. 